\title{
Taxa de cesariana nas primigestas atendidas numa maternidade pública com assistência humanizada no município de Juiz de Fora - MG
}

Vívian Campos Damasceno*
Leonardo Pandolfi Caliman**
Nathália Cristina Mezzonato Machado**
Ana Beatriz Clemente Gonçalves ${ }^{* * *}$
Leonardo César Mendes de Miranda****

\section{RESUMO}

Nos últimos tempos, o Brasil tem vivenciado uma mudança de paradigma na forma de nascer. A elevada taxa de cesarianas tem demonstrado a banalização desse procedimento. Na tentativa de mudar essa realidade, o Ministério da Saúde instituiu em todo o território brasileiro o Programa de Humanização no Pré-natal e Nascimento. Com o objetivo de verificar a frequência de parto cesáreo e as principais indicações de parto operatório no momento da internação nas primigestas atendidas na Maternidade Viva Vida de Juiz de Fora MG, foi realizado um estudo retrospectivo, baseado na análise do prontuário das primigestas internadas para parto na maternidade supracitada, durante o período de janeiro de 2013 a dezembro de 2014, obtendo um total de 811 primigestas. Foram investigados o perfil epidemiológico, risco pré-natal, motivo de internação, tipo de parto e as principais indicações de parto operatório no momento da internação. O parto cesáreo ocorreu em $40,0 \%$ das primigestas e os principais motivos que levaram à indicação de parto operatório no momento da internação foram as distócias $(37,8 \%)$ e os distúrbios hipertensivos maternos $(35,0 \%)$. O presente estudo concluiu que a taxa de cesariana encontrada foi semelhante à média da rede pública brasileira, sendo que a maioria das indicações de parto operatório no momento da internação permeavam as indicações relativas e absolutas preconizadas pelo Ministério da Saúde.

\section{Palavras-chave: Primigestas. Parto operatório. Parto vaginal. Humanização.}

\section{INTRODUÇÃO}

Nos últimos tempos, o Brasil tem vivenciado uma mudança de paradigma na forma de nascer (BRASIL, 2015). O nascimento, que historicamente é um evento natural e cheio de significados culturais, tem se tornado cada vez mais um objeto de medicalização (BRASIL, 2001; BRASIL, 2016). Desse modo, as operações cesarianas passaram a ser a via de parto mais comum, alcançando o percentual de $85 \%$ dos partos realizados na rede privada de saúde e $40 \%$ na rede pública (BRASIL, 2015).

Essa frequência elevada demonstra a banalização do procedimento, o qual passou a ser considerado simples e isento de possíveis complicações (MORAES; GOLDENBERG, 2001; SAKAE; FREITAS; d'ORSI, 2009). Entretanto, a operação cesariana, quando realizada sob indicações médicas específicas, é um procedimento cirúrgico fundamental para garantir a saúde materna e infantil (BARBOSA et al., 2003; WEIDLE et al., 2014; BRASIL, 2015; BRASIL, 2016).

Em 2015, após análise de evidências científicas, a Organização Mundial de Saúde (OMS), concluiu que taxa de cesariana superior a $10 \%$ não implica em redução da mortalidade materna, perinatal ou neonatal, sendo considerada aceitável uma taxa entre 10 a 15\%. Levando em conta a população obstétrica brasileira atual, que é composta por um elevado número de mulheres com cesárea prévia, estima-se que a taxa de referência esteja em torno de $29 \%$. Todavia, ao se analisar as taxas institucionais separadamente, é necessário que se observe o grau de complexidade da

\footnotetext{
*Hospital da Santa Casa de Misericórdia de Belo Horizonte, Departamento de Ginecologia e Obstetrícia, Belo Horizonte, MG. E-mail: viviancdamasceno@gmail.com

**Fundação Hospitalar do Estado de Minas Gerais, Hospital Regional João Penido, Departamento de Ginecologia e Obstetrícia, Juiz de Fora, MG.

***Universidade Presidente Antônio Carlos, Faculdade de Medicina de Juiz de Fora, Juiz de Fora, MG.

****Empresa Brasileira de Serviços Hospitalares, Hospital Universitário da Universidade Federal de Juiz de Fora, Departamento de Anestesiologia, Juiz de Fora, MG
} 
assistência fornecida por cada instituição (BRASIL, 2001).

$\mathrm{Na}$ tentativa de mudar essa realidade, na qual a segurança do parto normal frente ao cirúrgico vem sendo questionada, o Ministério da Saúde no ano de 2000 instituiu em todo o território brasileiro, através da Portaria/GM no 569, de 1/6/2000, o Programa de Humanização no Pré-natal e Nascimento (PHPN). O objetivo deste Programa é assegurar a melhoria do acesso, da cobertura e da qualidade do acompanhamento pré-natal, da assistência ao parto e puerpério às gestantes e ao recém-nascido, na perspectiva dos direitos de cidadania (BRASIL, 2002).

A humanização se caracteriza por dois aspectos fundamentais. $\mathrm{O}$ primeiro, no que tange ao direito da mulher e de seus familiares, bem como do recémnascido, de serem recebidos com dignidade pelas unidades de saúde (BRASIL, 2002; PEREIRA et al., 2007). Para isso, é necessária uma postura ética e solidária por parte da equipe de saúde e a organização da instituição no intuito de promover um ambiente acolhedor. $O$ segundo relaciona-se à utilização de medidas e procedimentos reconhecidamente benéficos para o acompanhamento do parto e do nascimento, evitando práticas intervencionistas desnecessárias (BRASIL, 2002; BRASIL, 2016).

Na literatura, poucos estudos foram descritos sobre o desfecho da via de parto em maternidades públicas onde a prática da assistência humanizada ao parto tem sido implementada. Entretanto, mostraram taxas de cesarianas inferiores à média brasileira. Paim et al. (2008) encontraram uma taxa de $25 \%$ ao analisarem cesárea primária para gestações de alto risco em um hospital referência de Porto Alegre. Já Silva et al. (2008), ao analisarem as estratégias de incentivo ao parto normal em um centro de parto normal (CPN), com atendimento apenas para gestantes de risco habitual, encontraram uma taxa de $21 \%$.

Um estudo realizado por Soares et al. (2017) em um CPN, constatou que as gestantes se beneficiam da assistência humanizada ao parto e nascimento preconizado pela OMS. Entretanto, estudos qualitativos mostram que as equipes de saúde ainda apresentam dificuldades para incorporar as práticas da assistência humanizada ao parto em seu cotidiano, demonstrando a necessidade de melhor capacitação da equipe, e que muitas gestantes e acompanhantes ainda desconhecem os seus direitos (REIS; PATRÍCIO, 2005; BUSANELLO et al., 2011; NAGAHAMA; SANTIAGO, 2011; SILVA; BARBIERI; FUSTINONI, 2011).

Dessa forma, o objetivo do presente estudo foi verificar a frequência de parto cesáreo nas primigestas atendidas na Maternidade Viva Vida de Juiz de Fora - MG e apontar os principais motivos relacionados à indicação de parto operatório no momento da internação.

\section{Material e métodos}

Foi realizado um estudo retrospectivo e descritivo por meio da análise de dados de prontuários das pacientes primigestas, que foram internadas para realização de parto, no período compreendido entre janeiro de 2013 a dezembro de 2014, na Maternidade Viva Vida do Hospital Regional João Penido FHEMIG, em Juiz de Fora - Minas Gerais.

A Maternidade Viva Vida foi estruturada com foco na assistência humanizada às gestantes e puérperas, tendo sido a pioneira na implantação do quarto PPP (pré-parto, parto e puerpério ou pós-parto) e, atualmente, a unidade é referência na região para atendimentos de alto risco.

O protocolo de pesquisa utilizado consta dos seguintes itens: perfil epidemiológico, risco pré-natal, motivo da internação, tipo de parto e principais indicações de parto operatório no momento da internação. Como critérios de inclusão tivemos todas as pacientes primigestas que foram internadas para realização de parto, no período mencionado acima, e como critério de exclusão as pacientes não primigestas. Esse estudo apresentou risco mínimo para as pacientes, uma vez que se limitou à revisão de prontuários.

Foram analisados todos os prontuários das pacientes que cumpriram os critérios de inclusão, totalizando 811 primigestas. Os dados foram armazenados no programa Access 20.0, Microsoft ${ }^{\circledR}$ Corporation, USA. Para a análise estatística foi utilizado o programa estatístico SPSS 20.0 (SPSS Inc, CDC, USA). Foram utilizados métodos descritivos para as variáveis estudadas e verificada a frequência das variáveis selecionadas. Após análise quantitativa dos dados, esses foram agrupados e apresentados em valores percentuais em tabelas.

No estudo foram observados os critérios preconizados na Resolução 466/12 do Conselho Nacional de Saúde (CNS), que regulamenta as normas éticas para pesquisa com seres humanos no Brasil, garantindo o sigilo das informações coletadas quanto ao nome das pacientes e seus dados pessoais.

O projeto foi cadastrado na Plataforma Brasil e aprovado pelo Comitê de Ética e Pesquisa da FHEMIG no parecer consubstanciado de $\mathrm{n}^{\circ} 1.343 .366$.

\section{Resultados}

O cisto periapical é uma entidade patológica deNo presente estudo retrospectivo, em relação às características sociodemográficas, das 811 primigestas internadas para parto na Maternidade Viva Vida de Juiz de Fora-MG, no período citado, verificou-se que grande parte das primigestas era solteira $(60,5 \%)$, com idade entre 19 e 34 anos (67,6\%), apresentava uma ocupação não remunerada $(49,9 \%)$ e ter concluído até o ensino médio $(56,1 \%)$ (Tabela 1$)$. 
TABELA 1

Perfil epidemiológico das primigestas atendidas para parto na

Maternidade Viva Vida de Juiz de Fora, MG (2013 - 2014).

\begin{tabular}{|c|c|c|c|c|c|}
\hline & $\begin{array}{c}\mathrm{n} \\
811\end{array}$ & $\%$ & & $\begin{array}{c}\mathrm{n} \\
811\end{array}$ & $\%$ \\
\hline Idade & & & Nível de instrução & & \\
\hline $10-18$ anos & 215 & 26,5 & Analfabeto & 1 & 0,1 \\
\hline $19-34$ anos & 548 & 67,6 & Fundamental & 217 & 26,8 \\
\hline \multirow[t]{3}{*}{$35-44$ anos } & 48 & 5,9 & Ensino médio & 455 & 56,1 \\
\hline & & & Ensino superior & 90 & 11,1 \\
\hline & & & Sem informação & 48 & 5,9 \\
\hline Estado civil & & & Profissão & & \\
\hline Solteira & 491 & 60,5 & Ocupação remunerada & 314 & 38,7 \\
\hline Casada & 223 & 27,5 & Do lar & 301 & 37,1 \\
\hline União estável & 61 & 7,5 & Estudante & 104 & 12,8 \\
\hline Outro & 11 & 1,4 & Sem informação & 92 & 11,3 \\
\hline Sem informação & 25 & 3,1 & & & \\
\hline
\end{tabular}

Fonte: Os autores.

No que se refere ao motivo de internação, houve prevalência do trabalho de parto espontâneo (61\%), sendo $52,5 \%$ a termo e $8,5 \%$ prematuro, seguido da indicação de indução $(21,2 \%)$ e de parto operatório $(17,8 \%)$. O desfecho da via de parto que predominou foi o parto vaginal (60\%), sendo que índices maiores foram encontrados nas primigestas que internaram por trabalho de parto prematuro $(89,8 \%$ ) (Tabela 2$)$.
TABELA 2

Motivo de internação das primigestas atendidas para parto na Maternidade Viva Vida de Juiz de Fora, MG (2013 - 2014).

\begin{tabular}{ccc}
\hline & $\mathrm{n}$ & $\%$ \\
\hline Motivo de internação & 811 & \\
Trabalho de parto a termo & 426 & 52,5 \\
Trabalho de parto prematuro & 69 & 8,5 \\
Indução & 172 & 21,2 \\
Parto operatório & 144 & 17,8 \\
\hline
\end{tabular}

Fonte: Os autores.

Das 144 primigestas que internaram para parto operatório, a maioria tinha como indicação a presença de distócias (qualquer problema de origem materna ou fetal que dificulte ou impeça o parto) (37,8\%), sendo as mais comuns por anormalidade de apresentação/ posição $(16,8 \%)$, por colo desfavorável à indução $(11,9 \%)$ e por desproporção fetopélvica (6,3\%). Em seguida, observaram-se os distúrbios hipertensivos maternos $(35,0 \%)$, tais como a pré-eclâmpsia grave $(22,4 \%)$ e a síndrome da complicação da eclâmpsia caracterizada por hemólise, enzimas hepáticas elevadas e plaquetopenia (HELLP) (6,3\%) (Tabela 3).

\section{TABELA 3}

Indicações de parto operatório no momento da internação nas primigestas internadas para parto na Maternidade Viva Vida de Juiz de Fora, MG (2013 - 2014).

\begin{tabular}{|c|c|c|c|c|c|}
\hline & $\mathrm{n}$ & $\%$ & & \multirow{2}{*}{$\begin{array}{c}\mathrm{n} \\
144\end{array}$} & \multirow[t]{2}{*}{$\%$} \\
\hline & \multicolumn{3}{|l|}{144} & & \\
\hline Distócias & & & \multicolumn{3}{|l|}{ Anormalidades cardíaca / circulatória fetal } \\
\hline Anormalidade de apresentação / posição & 24 & 16,8 & Anormalidades no doppler fetal & 14 & 9,8 \\
\hline Colo desfavorável à indução & 17 & 11,9 & Bradicardia fetal & 4 & 2,8 \\
\hline Desproporção fetopélvica & 9 & 6,3 & Taquicardia fetal & 1 & 0,7 \\
\hline Anormalidade dos tecidos moles do trato reprodutivo & 3 & 3,5 & Arritmia cardíaca fetal & 1 & 0,7 \\
\hline Discinesia uterina & 1 & 2,8 & & & \\
\hline Distúrbios hipertensivos materno & & & Distúrbios placentários & & \\
\hline Pré-eclâmpsia grave & 32 & 22,4 & Descolamento prematuro de placenta & 5 & 3,5 \\
\hline Síndrome HELLP & 9 & 6,3 & Placenta prévia & 3 & 2,1 \\
\hline Iminência de eclâmpsia & 5 & 3,5 & Síndrome da transfusão placentária & 1 & 0,7 \\
\hline Eclâmpsia & 4 & 2,8 & & & \\
\hline Anormalidades na formação / crescimento fetal & & & Outras & & \\
\hline Crescimento intrauterino restrito - CIUR & 25 & 17,5 & Gestação gemelar & 8 & 5,6 \\
\hline Malformação fetal & 14 & 9,8 & Primigesta idosa (feto precioso) & 3 & 2,1 \\
\hline Macrossomia fetal & 9 & 6,3 & Cesariana eletiva & 2 & 1,4 \\
\hline \multirow[t]{2}{*}{ Anormalidade no desenvolvimento fetal } & 1 & 0,7 & Intercorrências clínicas & 2 & 1,4 \\
\hline & & & Distúrbio mental materno & 1 & 0,7 \\
\hline Distúrbios do volume do líquido amniótico & & & Gestante HIV com carga viral desconhecida & 1 & 0,7 \\
\hline Oligodrâmnio & 15 & 10,5 & & & \\
\hline Adrâmnia & 3 & 2,1 & & & \\
\hline Polidrâmnio & 2 & 1,4 & & & \\
\hline
\end{tabular}

Fonte: Os autores. 
Ressalta-se que algumas primigestas apresentavam em seus prontuários mais de uma indicação de parto operatório, o que explica o número de indicações maior do que o número de primigestas que foram internadas para parto operatório.

\section{Discussão}

Em relação ao perfil sociodemográfico das primigestas avaliadas, observou-se que essas eram relativamente novas, sendo que $26,5 \%$ eram adolescentes. A maioria apresentou ser solteira $(60,5 \%)$, com ocupação não remunerada $(49,9 \%)$ e ter concluído até o ensino médio (56,1\%). Para Sanches, Mamede e Vivancos (2012), a constatação de um número reduzido de mulheres exercendo uma ocupação remunerada contrapõe-se a tendência de sua crescente inserção no mercado de trabalho. Acreditase que o alto percentual de mulheres exercendo uma ocupação não remunerada esteja relacionado, além da idade, com a baixa escolaridade verificada.

Neste estudo foram analisados 811 prontuários, observando-se que $60 \%$ dos partos evoluíram pela via vaginal e $40 \%$ pela via cirúrgica. Entretanto, o índice de parto cesáreo ainda se encontra longe do ideal proposto pela OMS, em 2015, a qual considera como taxa aceitável valores de até 15\%. Estudo semelhante realizado por Campana e Pelloso (2007), em um hospital público de referência para gestação de alto risco, encontrou uma taxa de parto operatório equivalente a 45,3\%. Em contrapartida, Paim et al. (2008), ao analisarem as taxas de cesárea primária em um hospital de Porto Alegre, também de referência para gestação de alto risco, encontraram uma taxa de $25 \%$.

Medeiros et al. (2016) analisaram a assistência em um hospital de ensino de Cuiabá, referência em alto risco, com unidades de pré-parto, parto e puerpério (PPP), observou uma redução superior a $10 \%$ na taxa de parto cesáreo após um ano da inserção de enfermeiras obstetras. Esses dados reforçam a importância de se implementar as práticas humanizadas nas maternidades visando um menor desfecho de partos cesáreos.

Embora a maternidade campo de estudo seja referência para gestação de alto risco, os pré-natais de alto risco corresponderam a apenas 23,8\%. Valores esses inferiores aos encontrados em um estudo semelhante realizado por Accetta et al. (2013), no qual $28 \%$ das primigestas possuíam pré-natal de alto risco e a taxa de parto cesáreo encontrada foi equivalente a 32,8\%. Como justificativa para o elevado índice de parto cesáreo, os autores mencionaram a possível influência das gestações de alto risco.
Não foi possível encontrar na literatura estudos que demonstrassem especificamente as principais indicações de parto operatório no momento da internação, ou seja, sem que fosse realizada a prova do trabalho de parto. Sendo assim, as comparações subsequentes serão feitas com autores que averiguaram as principais indicações de parto cesáreo de modo geral, por complicação de um trabalho de parto ou por complicação gestacional.

As distócias foram as causas mais comuns $(37,8 \%)$ de indicação de parto operatório no momento da internação, sendo a anormalidade de apresentação/ posição a mais frequente dentre elas. A cesariana pode ser indicada para gestantes com feto a termo em apresentação pélvica visando a redução da mortalidade perinatal e morbidade neonatal (BRASIL, 2015).

Em um estudo realizado em um CPN, Silva et al. (2008) encontraram uma taxa de 37\% de distócias nas indicações de parto cesáreo por complicação do trabalho de parto, sendo a discinesia uterina a principal delas. Outro estudo, ao verificar as indicações de cesárea em primigestas por complicação do trabalho de parto, encontrou uma taxa de $68 \%$ de distócias, sendo a desproporção fetopélvica a mais observada dentre elas (OLIVEIRA; CRUZ, 2010).

Como segunda causa mais comum de indicação de parto operatório no momento da internação encontrou-se os distúrbios hipertensivos maternos $(35,0 \%)$, sendo a pré-eclâmpsia grave a mais apontada dentre elas. Taxas inferiores de distúrbios hipertensivos maternos, equivalentes a $25,5 \%, 22,2 \%$ e 21,6\%, foram encontradas nos estudos de Campana e Pelloso (2007), Oliveira e Cruz (2010) e Accetta et al. (2013), respectivamente. Entretanto, no estudo realizado por Oliveira et al. (2016), os distúrbios hipertensivos apareceram como intercorrências maternas associadas ao parto cesáreo em 69,4\% das gestantes atendidas pela rede pública.

Cabe ressaltar que a pré-eclâmpsia grave não é uma indicação de cesariana per si, apesar de usualmente ser adotada, por ser um método rápido, sobretudo se existe deterioração da condição materna ou, nos casos de hipertensão grave, pelo temor de um descolamento prematuro de placenta ou lesão dos órgãos-alvo da gestante (XENAKIS et al., 1997; NASSAR et al., 1998). Em um estudo realizado por Linhares et al. (2014), a taxa de cesariana em gestantes com préeclâmpsia grave foi de 90,7\%. No presente estudo, a maioria das primigestas apresentou mais de uma indicação de parto operatório em seu prontuário, o que explica a presença de condições que isoladamente não sinalizariam a necessidade de um parto cesariano.

$\mathrm{O}$ estudo em questão possui limitações quanto à análise de dados, uma vez que os dados foram 
coletados de fontes secundárias (prontuários) passíveis de erros durante o preenchimento, abrangendo um período relativamente curto com amostra relativamente pequena. Por ser uma maternidade que apresenta características peculiares e uma clientela com determinado perfil sociodemográfico, torna-se questionável o grau de generalização para a população em geral. Porém, os dados obtidos podem servir como subsídios e ponto de partida para aprimoramento de outros estudos sobre o tema na região, bem como de retorno para a própria maternidade. Também servirá de alerta às autoridades na condução de políticas públicas da humanização do parto e nascimento.

\section{Conclusão}

O trabalho demonstrou que a maternidade campo de estudo, embora ofereça assistência humanizada às gestantes, ainda apresenta taxa de parto cesáreo semelhante à média da rede pública brasileira. Apesar do serviço ser referência para gestação de alto risco, houve uma taxa de cesariana acima da justificável, mostrando ser necessário repensar esta prática.

Entretanto, cabe ressaltar que grande parte das indicações de cesárea encontrada estava em consonância com as indicações relativas e absolutas preconizadas pelo Ministério da Saúde. Além disso, a maioria das primigestas submetidas à cesariana apresentou mais de uma indicação de parto operatório em seu prontuário, o que explica a presença de condições que isoladamente não sinalizariam a necessidade direta de uma condução cirúrgica.

Considerando que grande parte das cesarianas é justificada por cesáreas prévias e que as impressões sobre o processo de parturição são perpetuadas entre as mulheres, torna-se relevante o conhecimento da frequência de parto cesáreo em primigestas. É a gestante que passará pela experiência do parto pela primeira vez que poderá mudar o atual cenário do Brasil, no qual as operações cesarianas passaram a ser a via de parto mais comum.

\title{
Rate of caesarean section in the primigravidae attended at a public maternity hospital with humanized assistance in the city of Juiz de Fora - MG
}

\begin{abstract}
Lately, Brazil has experienced a switch-over paradigm in the form of birth. The high rate of caesarean sections has shown the trivialization of this procedure. In an attempt to change this reality, the Health Ministry established throughout the Brazilian territory the Program for Humanization of Prenatal and Birth. This study aimed to describe the frequency of caesarean section and the main indications of operative delivery at the moment of hospitalization in the primigravidae at the Maternidade Viva Vida of Juiz de Fora - MG. Thus, a retrospective study based on medical primigravidae records admitted to the aforementioned maternity during January 2013 to December 2014 was done, obtaining a total of 811 first pregnancy. It was investigated the epidemiological profile, prenatal risk, the reason for admission, the type of delivery and the main indications of operative delivery at the moment of hospitalization. The caesarean section represented $40 \%$ of deliveries and the main indications of operative delivery at the moment of hospitalization were dystocia (37.8\%) and hypertensive disorders $(35 \%)$. The present study concluded that the cesarean rate found was similar to the Brazilian public sector average, with the majority of indications of operative delivery at the moment of hospitalization permeating the relative and absolute indications recommended by the Ministry of Health.
\end{abstract}

Keywords: Primigravidae. Operative delivery. Vaginal delivery. Humanization.

\section{REFERÊNCIAS}

ACCETTTA, S. G. et al. Cesariana primária em nulíparas - fatores de risco em hospital público universitário. Revista HCPA, v. 33, n. 3, p. 198-204, set. 2013.

BARBOSA, G. P. et al. Parto cesáreo: quem o deseja? Em quais circunstâncias? Cadernos de Saúde Pública, v. 19, n. 6, p. 1611162, nov./dez. 2003.
BUSANELLO, J. et al. Atenção humanizada ao parto de adolescentes: análise das práticas desenvolvidas em um centro obstétrico. Revista Brasileira de Enfermagem, v.64, n.5, p.82432, set./out. 2011.

CAMPANA, H. C. R.; PELlOSO, S. M. Levantamento dos partos cesáreos realizados em um hospital universitário. Revista Eletrônica de Enfermagem, v.9, n.1, p.51-63, jan./abr. 2007.

HU Revista, Juiz de Fora, v. 43, n. 2, p. 121-126, abr./jun. 2017 
BRASIL. Ministério da Saúde. Portaria n 569, de 01 de junho de 2000 .

BRASIL. Ministério da Saúde. Secretaria de Políticas de Saúde. Área Técnica de Saúde da Mulher. Parto, aborto e puerpério: assistência humanizada à mulher. Brasília: Ministério da Saúde, 2001.

BRASIL. Ministério da Saúde. Secretária de Assistência à Saúde, Coordenação Materno-Infantil. Programa Humanização do parto: humanização no pré-natal e nascimento. Brasília: Ministério da Saúde, 2002. BRASIL. Ministério da Saúde (BR). Diretrizes de Atenção à Gestante: a operação Cesariana. Relatório de recomendação - Conitec. Brasília: Ministério da Saúde, 2015.

BRASIL. Ministério da Saúde (BR). Diretrizes Nacional de Assistência ao Parto Normal. Relatório de recomendação Conitec. Brasília: Ministério da Saúde, 2016.

LINHARES, J. J. et al. Fatores associados à via de parto em mulheres com pré-eclâmpsia. Revista Brasileira de Ginecologia e Obstetrícia, v. 36, n. 6, p. 259-263, mai. 2014.

MEDEIROS, R. M. K. et al. Cuidados humanizados: a inserção de enfermeiras obstétricas em um hospital de ensino. Revista Brasileira de Enfermagem, v. 69, n. 6, p. 1091-1098, nov./dez. 2016.

MORAES, M. S.; GOLDENBERG, P. Cesáreas: um perfil epidêmico. Cadernos de Saúde Pública, v. 17, n. 3, p. 509-519, mai./jun. 2001.

NAGAHAMA, E. E. I.; SANTIAGO, S. M. Parto humanizado e tipo de parto: avaliação da assistência oferecida pelo Sistema Único de Sáude em uma cidade do Sul do Brasil. Revista Brasileira de Saúde Materno Infantil, v. 11, n. 4, p. 415-425, out./dez. 2011.

NASSAR, A. H. et al. Severe reeclampsia remote from term: labor induction or elective cesarean delivery? American Journal of Obstetrics \& Gynecology, v. 179, n. 5, p. 1210-1213, nov. 1998.

OLIVEIRA, D. R.; CRUZ, M. K. P. Estudo das indicações de parto cesáreo em primigestas no município de Barbalha-Ceará. Revista Rene, v. 11, n. 3, p. 114-121. jul./set. 2010.
OLIVEIRA, R. R. et al. Factors associated to caesarean delivery in public and private health care systems. Revista da Escola de Enfermagem da USP, v. 50, n. 5, p. 733-740, sept./oct. 2016.

PAIM, A. D. et al. Taxa de cesárea primária. Revista HCPA, v.28, n.3, p.136-41, nov. 2008.

PEREIRA, A. L. F. et al. Pesquisa acadêmica sobre humanização do parto no Brasil: tendências e contribuições. Acta Paulista de Enfermagem, v. 20, n. 2, p. 205-215, jun. 2007.

REIS, A. E; PATRÍCIO, Z. M. Aplicação das ações preconizadas pelo Ministério da Saúde para o parto humanizado em um hospital de Santa Catarina. Ciência \& Saúde Coletiva, v. 10 (sup), p. 221230, mai. 2005.

SAKAE, T. M.; FREITAS, P. F.; D'ORSI, E. Fatores associados a taxas de cesárea em hospital universitário. Revista de Saúde Pública, v. 43, n. 3, p. 472-480, out. 2009.

SANCHES, N. C.; MAMEDE, F. V.; VIVANCOS, R. B. Z. Perfil das mulheres submetidas à cesariana e assistência obstétrica na maternidade pública em Ribeirão Preto. Texto Contexto Enfermagem, v. 21, n. 2, p. 418-426, abr./jun. 2012.

SILVA, F.M.B. et al. Centro de Parto Normal Como Estratégia de Incentivo ao Parto Normal: Estudo Descritivo. Enfermería Global, v.7, n. 14, p. 1-12, out. 2008.

SILVA, L. M.; BARBIERI, M.; FUSTINONI, S. M. Vivenciando a experiência da parturição em um modelo assistencial humanizado. Revista Brasileira de Enfermagem, v. 64, n. 1, p. 60-65, jan./ fev. 2011.

SOARES, Y. K. C. et al. Satisfação das puérperas atendidas em um centro de parto normal. Revista de Enfermagem da UFPE, Recife, v. 11 (supl.11), p. 4563-4573, nov. 2017.

WEIDLE, W. G. et al. Escolha da via de parto pela mulher: autonomia ou indução? Cadernos Saúde Coletiva, v. 22, n. 1, p. 46-53, mar. 2014.

XENAKIS, E. M. et al. Preeclampsia: is induction of labor more successful? American Journal of Obstetrics \& Gynecology, v. 89, n. 4, p. 600-603, apr. 1997.Oral Medicine, Oral Pathology, Oral Radiology, and Endodontics, v. 103, n.1, p. 16-18, jan. 2007. 